\title{
Avaliação de Musculaturas Respiratórias em lactentes por meio da eletromiografia de superfície: Uma revisão integrativa
}

\author{
M. Z. D’Arcadia ${ }^{1}$, I. M. Oliveira ${ }^{2}$, L. B. Matos $^{3}$, C. Z. Hallal ${ }^{4}$, A. A. Pereira ${ }^{5}$ e V. M. G. O. Azevedo ${ }^{6}$
}

1. Mestranda do Programa de Pós-Graduação em Ciências da Saúde, Faculdade de Medicina (FAMED) / Universidade Federal de Uberlândia (UFU). Uberlândia, MG, Brasil.

2. Pós doutoranda, UFU / Faculdade de Engenharia Elétrica (FEELT). Uberlândia, MG, Brasil.

3. Especialista em Fisioterapia Intensiva Neonatal e Pediátrica, Universidade Federal do Triângulo Mineiro (UFTM). Uberaba, MG, Brasil.

4. Doutora, Faculdade de Educação Física e Fisioterapia (FAEFI) /UFU, pós-graduação FAMED/UFU. Uberlândia, Minas Gerais, Brasil

5. Doutor, UFU / FEELT. Uberlândia, Minas Gerais, Brasil

6. Doutora, FAEFI/UFU, pós-graduação FAMED /UFU. Uberlândia, Minas Gerais, Brasil e-mail:marcadiazu@hotmail.com

\begin{abstract}
Resumo: Lactentes com doenças pulmonar crônica, apresentam um trabalho dos músculos acessórios da respiração aumentado para vencer a resistência do fluxo de ar que está comprometida, resultando na alteração da mecânica respiratória. Testes de função pulmonar são padronizados e realizados para a avaliação dessas alterações. Em lactentes existe uma grande dificuldade para realização, quando em respiração espontânea. Acreditamos que a eletromiografia (EMG) seja um método eficaz e de baixo custo para identificar tais alterações. Este estudo objetiva encontrar as evidências sobre avaliação de musculaturas respiratórias em lactentes por meio da eletromiografia. Realizou-se uma revisão integrativa nas bases de dados PUBMED, no período de 2006 a 2016 e foram selecionados três artigos. A análise destes indica que a EMG é viável e replicável em prematuros em ventilação mecânica, porém muito criticada pela possibilidade de contaminação da atividade mioelétrica por outros músculos. Ressalta-se a importância de mais estudos e experimentos neste tema.
\end{abstract}

Palavras-chave: Eletromiografia de superfície, Testes de função Respiratória, Respiração.

Abstract: Infants with chronic lung diseases, present a work of accessory muscles of respiration increased to overcome the resistance of air flow that is committed, resulting in the alteration of respiratory mechanics. Pulmonary function tests are standardized and carried out for the evaluation of these changes. In infants, there is a great difficulty to perform, when in spontaneous breathing. We believe it to electromyography (EMG) is an effective and inexpensive method to identify such changes. This study aims to find the evidence on muscles respiratory assessment in infants by electromyography. In an integrative review in the databases PUBMED, in the period from 2006 to 2016, three articles were selected. The analysis of these articles indicates that EMG is viable and replicable in premature infants on mechanical ventilation, but much criticized for the possibility of contamination of the myoelectric activity by other muscles. It suggests the importance of further studies and experiments on this topic.

Keywords: Surface electromyography, Respiratory function testes, Breathing.

\section{Introdução}

Crianças portadoras de doenças pulmonares crônicas apresentam uma alteração da mecânica respiratória, resultante do trabalho dos músculos acessórios da respiração aumentado para vencer a resistência do fluxo de ar que está comprometida. ${ }^{1,2,3} \mathrm{O}$ uso excessivo dessa musculatura respiratória pode ocasionar não somente alterações na mecânica respiratória como também na postura. ${ }^{4}$

A avaliação da atividade mioelétrica dos músculos respiratórios, é capaz de fornecer informações detalhadas sobre o comportamento neuromuscular e identificar os comportamentos motores anormais, tanto da caixa torácica como da mecânica respiratória. Para a avaliação dessas alterações podemos utilizar a eletromiografia, um método eficaz e de baixo custo., ${ }^{4,5}$

Além disso, pequenas alterações no recrutamento motor são particularmente difíceis de detectar com a palpação ou inspeção visual quando o paciente está contraindo a musculatura respiratória acessória em alto ou baixo nível de força, sendo assim, somente através da eletromiografia pode-se detectar súbitas alterações na ativação muscular. ${ }^{7}$

Após detecção dessas alterações, será possível indicar o tratamento precoce, preventivo e direcionado amenizando as alterações da mecânica respiratória, resultando em uma melhora da qualidade de vida dos 
indivíduos. Este estudo objetiva avaliar as evidências que avaliam musculaturas respiratórias em lactentes, por meio da eletromiografia de superfície.

\section{Materiais e métodos}

Realizou-se uma busca na literatura, utilizandose a estratégia metodológica de Revisão Integrativa, que consiste na análise de pesquisas relevantes e na síntese do conhecimento sobre um determinado assunto. A pergunta que orientou o estudo foi: "Quais são as evidências sobre a avaliação de musculaturas respiratórias de lactentes, por meio da sEMG? ". Foi consultado na base de dados eletrônicas PubMed. Os critérios de seleção foram: artigos randomizados publicados nos idiomas português, inglês e espanhol, dos últimos 10 anos, idade de recém-nascido até 23 meses de vida, humanos. De exclusão foram: publicações em outros idiomas, indisponibilidade de recuperar a publicação na íntegra por meio de comutação e inadequação ao objeto de estudo. O levantamento foi realizado com os Descritores em Ciências da Saúde (DeCS), combinados da seguinte forma: Surface Electromyography AND Respiratory function testes; Surface Electromyography AND Breathing, respectivamente com 14 e 33 registros, totalizando 47. A pesquisa foi realizada em 02 de agosto de 2016. Os 47 resumos foram lidos autonomamente por dois pesquisadores, para identificar aqueles que atendiam aos critérios de seleção. Três publicações foram selecionadas e as demais excluídas, por não atenderem aos critérios estabelecidos.

\section{Resultados}

Foram selecionados três artigos sobre experimentos randomizados. A síntese desses estudos encontra-se na Tabela 1.

Tabela 1 - Descrição dos artigos selecionados segundo o objetivo, a amostra e os métodos utilizados, principais resultados encontrados e conclusões. 2006 - 2016.

\begin{tabular}{|c|c|c|c|c|c|}
\hline \# & dr & $\Rightarrow$ & - & 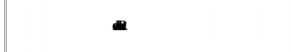 & क \\
\hline 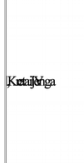 & 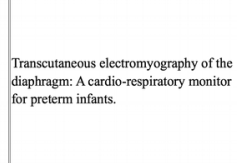 & 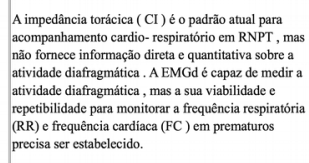 & 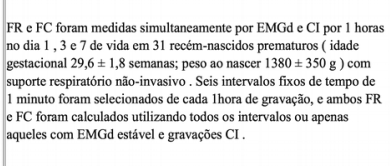 & 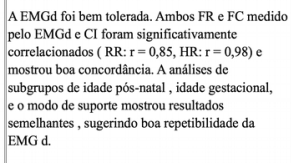 & 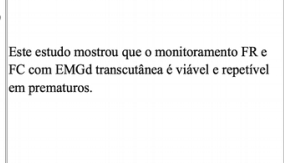 \\
\hline |ritaten, & 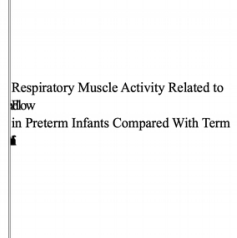 & 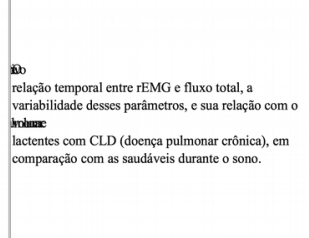 & 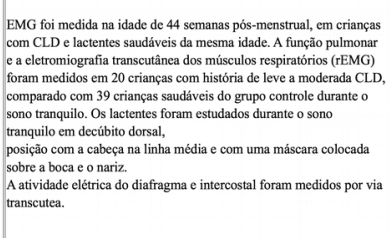 & 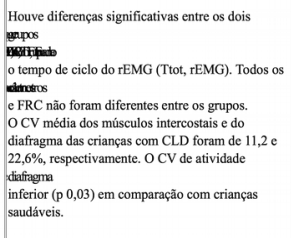 & 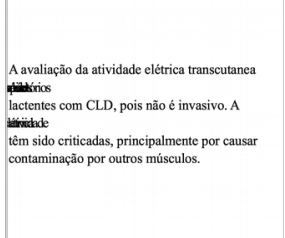 \\
\hline Hitutan, & $\begin{array}{l}\text { Relativiv impact of respiratory muscle } \\
\text { activity on tidal flow and end expiratory } \\
\text { moume }\end{array}$ & 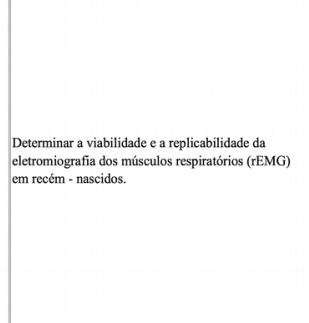 & 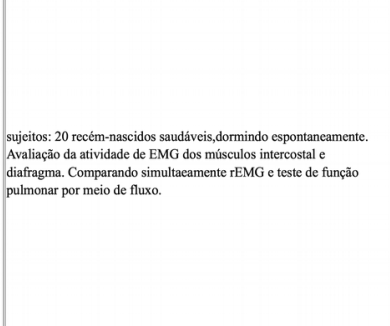 & 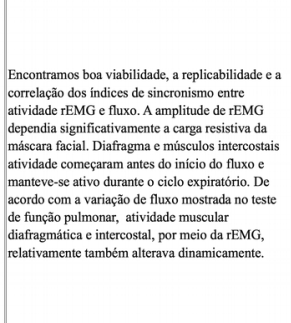 & 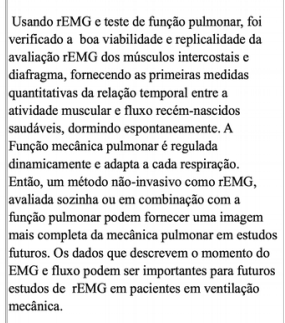 \\
\hline
\end{tabular}

\section{Discussão}

\section{Qualidade metodológica dos artigos}

Todos os artigos selecionados para esta revisão eram experimentais e apresentavam um grupo controle, o que se constitui num cuidado metodológico relevante para a avaliação de musculaturas respiratórias em lactentes, por meio da eletromiografia de superfície.

\section{Modo de aplicação da EMG}

Em todos os artigos foi realizado a eletromiografia de superfície, um método não invasivo. A EMG foi realizada nos músculos respiratórios diafragma e intercostal. Avaliação realizada em recémnascidos sob ventilação mecânica sem sedação. Dois 
artigos realizam sincronicamente a analisa da EMG com teste de função pulmonar. E um deles avalia a EMG em conjunto com a impedância torácica. Em todos os três artigos selecionados, mostrou que a eletromiografia de superfície é viável e replicável em prematuros sob ventilação mecânica, porém muito criticada pela possibilidade de contaminação da atividade mioelétrica por outros músculos.

$\mathrm{O}$ monitoramento da frequência respiratória (FR) e frequência cardíaca (FC) com EMG é viável e replicável em prematuros. ${ }^{8}$

A combinação de testes de função pulmonar e eletromiografia dos músculos respiratórios (rEMG) em lactentes saudáveis, sugerindo que a interação dos músculos respiratórios e mecânica pulmonar são ativamente controlados pela respiração. E a medição simultânea dos testes de função pulmonar e parâmetros da rEMG potencializam a análise da mecânica respiratória na doença. Segundo os autores, os bebês com doença pulmonar crônica tentam manter um volume pulmonar relativamente normal, alterando os padrões e a dinâmica respiratória e elevando o volume pulmonar. Estes mecanismos podem ser detectados tanto por diferenças na relação de tempo entre rEMG como por alteração de fluxo e volume pulmonar nos testes de função pulmonar. ${ }^{9}$

A rEMG combinada com os testes de função pulmonar apresentou boa viabilidade e replicabilidade na mensuração da atividade muscular dos intercostais e diafragma, uma vez que fornece as medidas quantitativas da relação temporal entre a atividade muscular e o deslocamento do fluxo aéreo em recémnascidos saudáveis dormindo espontaneamente. A função da mecânica pulmonar é regulada dinamicamente e adapta-se a cada respiração. Então, o rEMG, sendo um método não invasivo, medido sozinho ou em combinação com os testes de função pulmonar, pode fornecer um quadro mais abrangente da mecânica pulmonar. ${ }^{10}$

É possível afirmar que a EMG é viável e replicável em prematuros em ventilação mecânica, porém com critica pela possibilidade de contaminação da atividade mioelétrica por outros músculos.

\section{Referências}

[1] Decramer, M. Hyperinflation and respiratory muscle intraction. Eur Respir J. 1997;10:93441.10 .

[2] De Troyer, A. Effect of hyperinflation the diaphragm.Eur Respir J.1997;10(4):708-13.

[3] Freire, A L G; Paes, F. J. V ; Amorim, C ; Chiavegato, L. D . Avaliação da atividade dos músculos acessórios da respiração em crianças asmáticas no período assintomático.. Revista Terapia Manual , v. 10, p. 88-95, 2012

[4] Hruska, R. J. Jr. Influences of dysfunctional respiratory mechanics on orofacial pain. Dent Clin North Am, 41 (2):211-27, Apr 1997.
[5] Ribeiro, E.C., Marchiori, S.C., Silva, A. M. Eletromiografia dos músculos esternocleidomastóideo e trapézio em crianças respiradoras bucais e nasais durante correção postural. Arq Int Otorrinolaringol. 2003;7: 139.

[6] Turner, M.O., Noertjojo, K., Sverre V. et al., Risk factors for near-fatal asthma. Am J Respir Crit Care Med 1998;157(6):1804-09.

[7] Blinder-Macleod, A. S.; Wolf, L. Ssteven. Neurophysiological Eletromyographic Factors for Neuromotor Disturbances. In: Biofeedback, 2th ed., Baltimore; Willians and Wilkins, 1982: 1736.

[8] Kraaijenga, J. V., Hutten, G. J., de Jongh, F. H. and van Kaam, A. H. (2015), Transcutaneous electromyography of the diaphragm: A cardiorespiratory monitor for preterm infants. Pediatr Pulmonol.,50:889-895.doi:10.1002/ppul.23116

[9] Hutten G. J. , Eykern L. A., Latzin P., Thanrin C., Aalderen W. M., Frey U. Respiratory Muscle Activity Related to Flow and Lung Volume in Preterm Infants Compared with Term Infants. International Pediatric Research 68: 339-343, 2010.

[10] Hutten G.J., Ekern L.A., Latzin P., Kyburz M., Aalderen W. M., Frey U. Relative Impact of Respiratory Muscle Activity on Tidal Flow and End Expiratory Volume in Healthy Neonates. Pediatric Pulmonology 43: 882-891, 2008. 